\title{
Optical distortion compensation in visual measurement with a new Depth From Defocus method
}

\author{
W. Sulej, ${ }^{* 1}$ K. Murawski, ${ }^{1}$ T. Pustelny ${ }^{2}$ \\ ${ }^{1}$ Institute of Teleinformatics and Automation, Military University of Technology, Kaliskiego 2, 00-908 Warsaw, \\ ${ }^{2}$ Department of Optoelectronics, Silesian University of Technology, Krzywoustego 2, 44-100 Gliwice
}

Received November 03, 2017; accepted December 02, 2017; published December 31, 2017

\begin{abstract}
The paper presents a new approach to compensate for the inaccuracy of shape mapping of the flaccid membrane used in the extracorporeal pneumatic heart assist pump. The original shape of the membrane was determined using a specially developed DFD type method. The major problems affecting the inaccuracy of mapping the membrane result from the phenomenon of optical distortion. The invented approach to compensate for optical distortion in the process of visual measurement as well as the results obtained were presented.
\end{abstract}

The authors are working on an unsolved problem in the world of determining the instantaneous volume of the blood chamber of the pulse-type heart assist pump. The extracorporeal pneumatic heart assist pump, being the object of our study, has a pneumatic chamber and a blood chamber separated by a flaccid membrane. The authors propose the solution where the momentary volume of the blood chamber will be determined instantaneously on the basis of the shape of the blood pumping membrane. As the shape of the flaccid membrane varies randomly, the problem of determining the volume of the artificial ventricle is a huge mathematical and numerical challenge.

A new innovative Depth From Defocus (DFD) method was developed for visual distance measurement [1]. In [2], it was used to determine the shape of the flaccid membrane of the extracorporeal pneumatic heart assist pump. The membrane is controlled by the gas forced into the pneumatic chamber. This is a continuation of the earlier research on the use of image processing and analytical techniques to determine the membrane shape and stroke volume of an artificial ventricle [3-7].

The essence of the used measurement method is to observe the surface of the membrane at a close distance with a motionless fixed-focus camera equipped with a wide-angle lens and to determine the shape of this membrane in 3-dimensional space on the basis of a one-shot image only. This method works due to markers arranged on the surface of the membrane from the pneumatic chamber side. During membrane movement the markers do not change their physical size. Their smaller or larger surface area visible in the image is related only to its proximity or distance from the camera optical sensor. The measurement method can take two forms of implementation. Until now the mapping and visualization

*E-mail: Wojciech.Sulej@wat.edu.pl of the membrane shape has been performed only in the virtual world - in pixels. Currently, works are carried out to determine the membrane shape in actual dimensions (in millimetres) - in the real world [8].

Membrane shape mapping in actual dimensions will enable to determine the stroke volume directly using a numerical integration method. This approach to visual measurement requires overcoming significant problems arising from membrane image optical distortions, which are formed in the optical path of the camera. During data analysis it was noticed that the major problems with the accuracy of membrane mapping result from optical distortion of the image [9]. Optical distortion is a deviation from the rectilinear projection when the straight lines in an observed scene are not straight in the image. In the study we can state the barrel distortion, Fig. 1a. This manifests itself by the fact that the markers of the same size and distance from the optical sensor, arranged further from the centre of optical axis of the camera, are smaller in the image than the markers located closer to the centre. It is the worst at the wide-angle end of the range. The flat surface of the membrane appears to be bulged upwards. The optical distortion phenomena could not be avoided due to the need of using the camera lens of a small focal length $f$ and a large viewing angle $\alpha$, in the study $f=1.8 \mathrm{~mm}$ and $\alpha=126^{\circ}$. It is necessary due to the short distance from the front of the camera lens to the surface of the membrane, which is approximately $20 \mathrm{~mm}$, and the membrane diameter of $70 \mathrm{~mm}$.

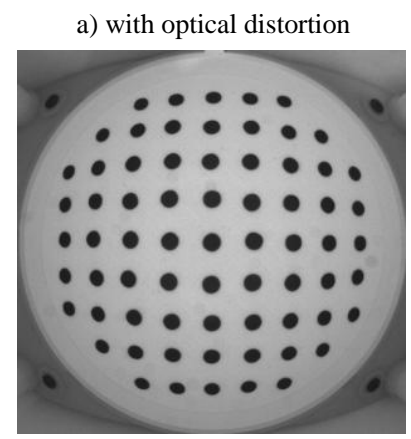

b) without optical distortion

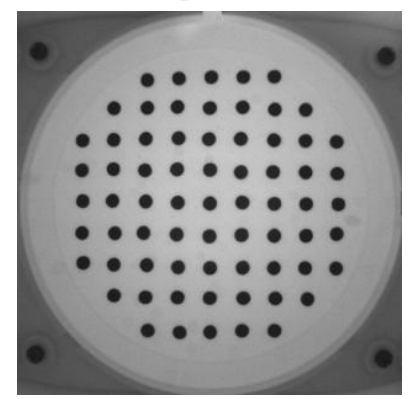

Fig. 1. Membrane view from the camera. 
The classic approach to eliminate optical distortions uses the Brown-Conrady model [10-12]. In this model the famous plumb line method was proposed. According to the model, for each pixel in the image, the $x$ and $y$ coordinates have new values $x_{u}$ and $y_{u}$ calculated on the basis of equations:

$$
\begin{aligned}
& x_{u}=x\left(1+\mathrm{k}_{1} \mathrm{r}^{2}+\mathrm{k}_{2} \mathrm{r}^{4}+\mathrm{k}_{3} \mathrm{r}^{6}\right)+\left[2 \mathrm{p}_{1} x y+\mathrm{p}_{2}\left(\mathrm{r}^{2}+2 x^{2}\right)\right], \\
& y_{u}=y\left(1+\mathrm{k}_{1} \mathrm{r}^{2}+\mathrm{k}_{2} \mathrm{r}^{4}+\mathrm{k}_{3} \mathrm{r}^{6}\right)+\left[\mathrm{p}_{1}\left(\mathrm{r}^{2}+2 y^{2}\right)+2 \mathrm{p}_{2} x y\right] .
\end{aligned}
$$

As can be seen, the calculation of the values of $x_{u}$ and $y_{u}$ requires the knowledge of the values of coefficients $\mathrm{k}_{1}, \mathrm{k}_{2}$, $\mathrm{k}_{3}$, as well as $\mathrm{p}_{1}$ and $\mathrm{p}_{2}$. They are determined by placing them in the field of the camera view of a chess pattern with known area sizes. Afterwards, a few or several images have to be acquired, changing the position of the pattern between recorded images. Characteristic points are determined for the acquired images and new mapping is calculated. As a result, it is possible to calculate the desirable parameters of Eqs. (1) and (2). These parameters for the given optical system are determined only once. The described mechanism of eliminating optical distortion is available for example in Matlab. The chess pattern used in the study had the width and height of the field equal to $10 \mathrm{~mm}$. During calibration, it was placed in the position of the membrane.

However, the classic approach does not provide correct results in combination with the invented DFD method. The problem does not lie in the part of the method that concerns the $x$ - and $y$-coordinates because they are determined directly, based on pixel coordinates of the image [8]. Thus, there is no loss of information after the conversion from the $x$ and $y$ coordinates of pixels in the image to $x_{u}$ and $y_{u}$ respectively if the classic approach is used in this part. However, the z-coordinate of each marker location in 3-dimensional space is determined using a blurred view of the marker [1]. The classical model does not take into account the $z$-coordinate; after conversion the information obtained from the blurring is lost. Thus, it was necessary to develop a different approach.

Since optical distortion cannot be eliminated from the image without any information loss, its influence on measurement results should be compensated. The innovative approach to compensate for optical distortion of the image of the membrane is based on the properties of the used measurement method. In the study, observing the tensioned flat membrane with recognizable markers, it was noted that in the absence of any observed distortion all markers should have the same surface area in the image as the central marker, Fig. 1b. Thus, knowing the surface area of any marker in the optically distorted image, we can calculate the difference of its surface area and the surface area of the central marker, expressing it as a percentage. The elimination of a measurement error over the entire image area required determining compensation coefficients for each possible location of the marker. The studies take into account the movement of the marker in the Z-axis and the coordinates $\mathrm{X}$ (width) and $Y$ (height) of the image.

Having the membrane with 49 markers in the study, the same number of compensation coefficients was obtained. Other coefficients were determined using interpolation. For this purpose, the function of a triangulation-based cubic interpolation of 2D scattered data was implemented in Matlab. The data of all markers were transmitted to the function. This resulted in the formation of a percentage chart of compensation coefficients $\mathrm{Z}=f(\mathrm{X}, \mathrm{Y})$, which determines the correction result for each possible location of the marker, Fig. 2. The shape of the resulting data grid resembles an elliptic paraboloid but with some apparent irregularities. Additionally, in the chart several ranges of compensation coefficient values have been highlighted by different colours depending on the distance of a possible marker location from the centre of the camera optical axis. In close proximity to the central marker (blue), the coefficient is about $0 \%$ and the surface area of markers in these locations does not have to be compensated. Near to the outline of a circular membrane (red) it grows to about $15 \%$ and then the compensation of the markers surface area is the greatest.

The values of compensation coefficients were saved in the form of a matrix with the same size as the image resolution in pixels, in the study $900 \times 900$. Having values $\mathrm{X}$ and $\mathrm{Y}$ from the image, we can treat them as the indices of this matrix. In the measurement the surface area of the marker after compensation is calculated automatically from Eq. (3), where $A_{z}$ is the surface area of the current marker and $A_{\text {zero }}$ is the surface area of the central marker on the level of the outline of a circular membrane obtained in the calibration process:

$$
A_{c}=A_{z}+A_{\text {zero }} * f(\mathrm{X}, \mathrm{Y})
$$

Only after this operation is the $z$-coordinate of each marker location in the 3-dimensional space determined using $A_{c}$ based on the transformation equations presented in [8].

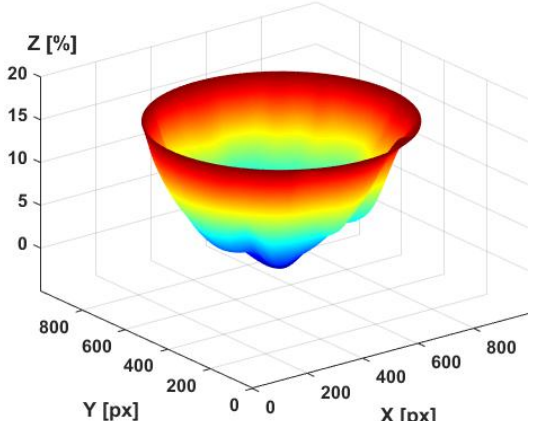

Fig. 2. Chart of compensation coefficients. 
a) with optical distortion

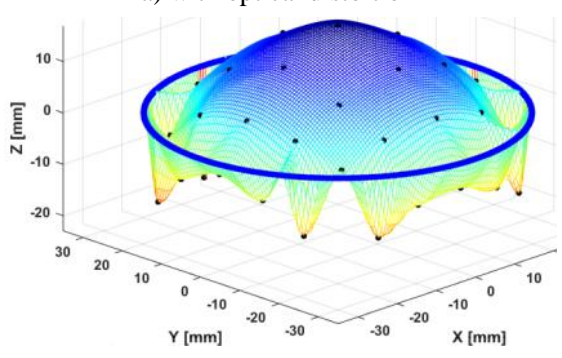

b) after elimination optical distortion

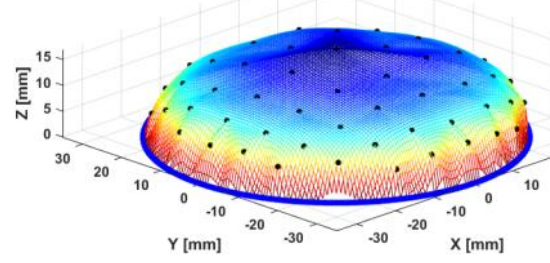

Convex state
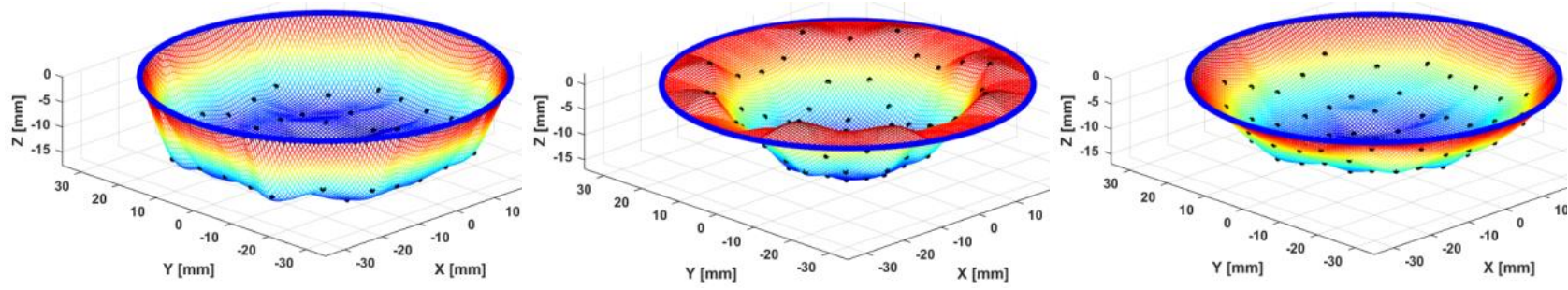

Concave state c) after invented compensation

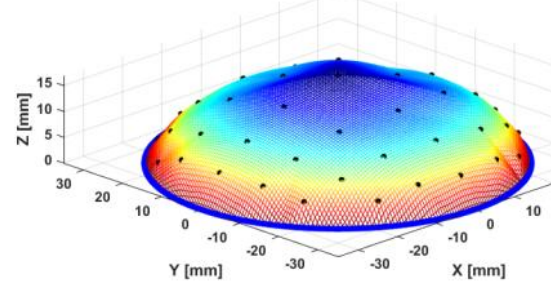

$\mathrm{Y}[\mathrm{mm}]$

Fig. 3. Meshes representing the membrane shape obtained on the basis of the measurements results for two extreme states ot the membrane.

While carrying out measurements using the invented DFD type method for determining the membrane shape in the actual dimensions without introducing any additions or amendments, high inaccuracy of shape mapping of the tested membrane was obtained, Fig. 3a. In the case of the extreme convex state of the membrane, Fig. 3a "Convex state", the shape of the projection is subject to the smallest error in the vicinity of the central marker. The biggest distortion was obtained for markers which location was close to the edge of the membrane. It is noted here that markers are located below the outline of a circular membrane, which does not exist in reality. With the extreme concave state of the membrane, Fig. 3a "Concave state", it assumes the shape resembling a more cubical than spherical cap.

The removal of optical distortion using the classic approach yielded only slightly better mapping results of the membrane shape, Fig. 3b. For the extreme convex state of the membrane, Fig. $3 \mathrm{~b}$ "Convex state", the elimination of optical distortion resulted in getting rid of the markers which were located below the outline of a circular membrane, but the regions located close to the membrane edge became too vertical. In the case of the extreme concave state of the membrane, Fig. $3 \mathrm{~b}$ "Concave state", the membrane shape mapping is correct for about half of the surface area counting from the central marker. Unfortunately, the regions located close to the membrane edge became too flat.

In the tested cases, only the invented method of optical distortion compensation used in the process of visual measurement radically improved the accuracy of membrane shape mapping, Fig. 3c. The membrane mesh takes on the correct shape - an almost regular spherical cap - both for the extreme convex state of the membrane, Fig. 3c "Convex state", and the extreme concave state of the membrane, Fig. 3c "Concave state". Evaluating the results was done by using the well-known mathematical description of the geometric dimensions of the spherical cap. It has been found that in both cases the difference between all of the measurement values and their reference values does not exceed 5\%. It is much better than about $10 \%$, which is generally accepted in the measurement of the stroke volume by cardiologists [3].

The developed new method of optical distortion compensation in the visual measurement allowed to achieve a significant accuracy improvement of membrane shape mapping of the extracorporeal pneumatic heart assist pump in the actual dimensions.

\section{References}

[1] K. Murawski, Method of measuring the distance using one camera, Patent Application No P.408076 (2014) - in Polish.

[2] K. Murawski, M. Murawska, T. Pustelny, The system and method of determining the shape of the membrane pneumatic pump of extracorporeal heart assist device, Patent Application No P.414104 (2015) - in Polish.

[3] M. Gawlikowski, T. Pustelny, R. Kustosz, Journal de Physique IV France 137, 73 (2006).

[4] K. Murawski, Acta Phys. Pol. A 128(1), 10 (2015).

[5] K. Murawski, Metrology and Measurement Systems 22(1), 69 (2015).

[6] K. Murawski, A. Arciuch, T. Pustelny, Phot. Lett. Poland 8, 2 (2016).

[7] L. Grad, K. Murawski, W. Sulej, Proc. SPIE 10455, 1045509 (2017).

[8] W. Sulej, K. Murawski, Proc. FedCSIS ACSIS 11, 675 (2017).

[9] W. Sulej, L. Grad, K. Murawski, Proc. SPIE 10455, 104550B (2017).

[10] D.C. Brown, Decentering distortion of lenses (1966).

[11] A.E. Conrady, Decentred Lens-Systems (1919).

[12] D.C. Brown, Close-Range Camera Calibration (1971). 